\title{
Autonomous Real-time Transmission Technology for Multiple Emergencies' Information
}

\author{
Fan WEI ${ }^{1, *}$, Hui-jing GE$^{1}$ and Xian-wei ZHANG ${ }^{1}$ \\ ${ }^{1}$ School of Computer Science, Xi'an Shiyou University, Shaanxi, P. R. China, 710065 \\ Email: fwei@xsyu.edu.cn, 1187092706@qq.com,xwzhang@xsyu.edu.cn
}

Keywords: Emergency, Real-time, Community

\begin{abstract}
Wireless Sensor Network(WSN) is widely used in Emergency Management System(EMS) to assure requirement of safety in current society. When emergency appears, real-timely transmitting emergency information should be assured. But this requirement can not be satisfied by conventional approaches which are based on static situations and centralized management. In this paper, autonomous real-time transmission technology is proposed to construct a special group of routers, called community which includes emergency transmission route for emergency information's transmission, and barrier which protects emergency information's transmission from interference of normal sensing information's transmission. Evaluation results indicate improvement of real-time property of multiple emergencies' information.
\end{abstract}

\section{Introduction}

A WSN[1] consists of a large number of sensor nodes, which are densely deployed in a region to sense environment or certain objects. Sensing information obtained by sensors is transmitted through the network to some certain terminals for further processing.

Nowadays, higher and higher safety of each industrial and social system is being required. Thus, Emergency Management System (EMS)[2] which is composed of many sensors, monitor stations and other hardware and software modules, is playing an important role in current society.

At EMS, dynamic situations such as system updating and expansion, appearance of emergency situation and failure of system equipment etc. happen anywhere and anytime. It is very difficult to predict them. When emergency happens, timely transmitting emergency information should be the most important point. But conventional approaches[3,4] are based on static situations and centralized management strategy. At dynamically changing situations, emergency information's real-time property can not be assured.

In order to adapt the dynamically changing situations, Autonomous Decentralized System (ADS)[5] was proposed. Each subsystem of ADS gathers information, autonomously judges and autonomously processes. Based on ADS concept, community is proposed. Community[6,7,12] is defined as a group that all members cooperate and coordinate with each other autonomously for the same objective. In this paper, to guarantee real-timely transmitting emergency information, autonomous real-time transmission technology is proposed. Transmission of emergency information is protected by proposed technology from interference[8] of normal sensing information's transmission. 


\section{System Architecture}

In this paper, Autonomous Community Wireless Sensor Network System (ACWSNS) is defined as a group composed of multiple wireless routers, include main route and barrier. When emergency happens, community is autonomous constructed. Emergency transmission route is only for transmitting emergency information. Barrier will protect the emergency information's transmission.

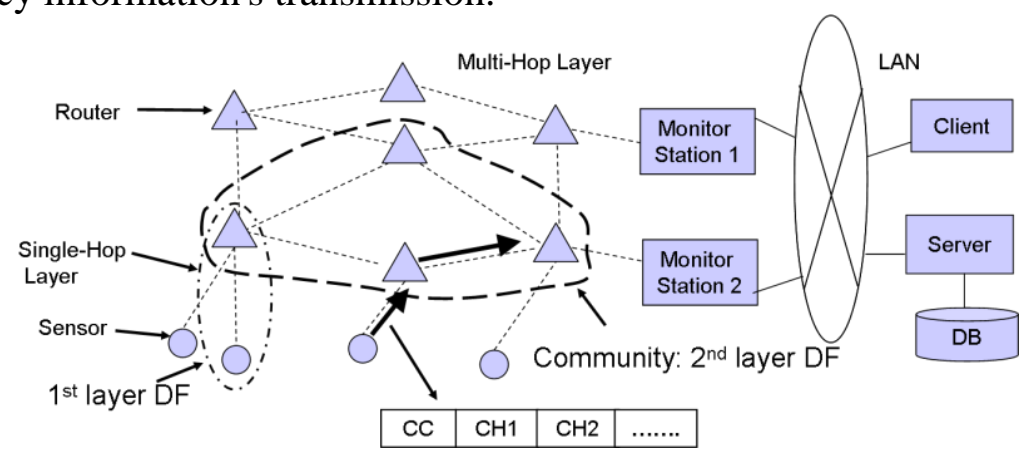

Figure 1. System Architecture

Fig.1 shows the ACWSNS's architecture via wireless sensors, routers[9] and multiple monitor stations. Monitor station connects with each other by wired communication. Connections between router and monitor station, router and router, router and sensor are wireless. Mesh network model is utilized. Each sensor will connect one router and send sensing information to router. Each router, connects with sensors and other routers, receives information from sensors and other routers, autonomously processes and forwards information via own judgment. In this architecture, sensors only sense and submit the sensing information to routers. Routers which have more battery and stronger transmission ability act as transmitters. Two layers Data Field(DF)[5] architecture is introduced. First layer DF is constructed via one sensor and its connected router and normally is fixed. Second layer DF is constructed by community members which are routers. Unlike permanent DF, this DF is temporary.

\section{Autonomous Real-time Transmission Technology}

\section{Autonomous Routing}

Object of autonomous routing is making each router to know which router to forward sensing information. In each router, there are 3 tables. First is neighbor table. Second is monitor station table. Third is own state table which includes connected monitor station, connected router and load.

As LAN is used for connection between monitor stations, thus each monitor station can periodically submit routing messages in network at same time.

$$
\begin{array}{|l|l|l|l|l|l|l|l|}
\hline \text { Routing } & \text { senderID } & \text { sourcelD } & \text { Msg Id } & \text { Ioad } & \text { hop } & \text { MS_ID } & \text { MS_load } \\
\hline
\end{array}
$$

Figure 2. Routing Message

When received a routing message as shown in Fig.2, firstly the router should check whether the monitor station id is existed in its own table. If yes, information of monitor station's load should be refreshed. Timer should be refreshed to incipient value. Otherwise, the new monitor station's information should be added to monitor station table. 
Second step is refreshing information in neighbor table. 1)Based on message id of routing message, check the sender id of routing message. 2) If the sender id did not exist in neighbor table, its information should be added to neighbor table. Otherwise, neighbor router's information should only be refreshed.

Third step is selecting connected router and monitor station. 1)Based on the hop part of routing message, select monitor station which has the least hops to the router. If hops are same, based on the information of monitor station's capacity, select monitor station which has most capacity. 2)Based on information of hops and capacity of each neighbor router, the neighbor which is connected to the selected monitor and has the most capacity should be selected..

Finally, the router should submit the routing message which includes its own information to neighbors. After these 4 steps of autonomous routing, each router could know its upper router.

\section{Autonomous Emergency Detection}

If emergency appears, corresponding sensor will firstly sense it. Therefore, each sensing information message which is generated by sensor should be given with $\mathrm{CC}$ of "Emergency".

\begin{tabular}{|l|l|l|l|l|l|}
\hline Detection & Upper & Sender & $\begin{array}{l}\text { Source } \\
\text { Routerr }\end{array}$ & $\begin{array}{l}\text { Source } \\
\text { Sensor }\end{array}$ & Msg Id \\
\hline
\end{tabular}

Figure 3. Emergency Detection Message

When one router receives sensing information message with CC of "Emergency" from connected sensor, it could be autonomously judged emergency has appeared. Router should do corresponding operations as following: 1) Change state from "Normal" to "Detection". 2) Generate emergency detection message shown in fig.3. "Source Sensor" field is the connected sensor which sensed emergency. "Source Router" and "Sender" fields are router's id. "Upper" field is its upper router's id. 3) Broadcast this generated message as shown in Fig.3 to all neighbor routers. This router is origin of emergency transmission route.

\section{Autonomous Emergency Transmission Route and Barrier Construction}

Because each router knows its "upper" router, it should forward the received emergency detection message to its "upper" router. As broadcasting characteristic of wireless communication, all neighbor routers could receive this message. The following will explain the sequence of route and barrier construction.

If one router received emergency detection message from its neighbor router, firstly it should check whether this message is from "lower" router by comparing its id with "Upper" field of message. If same, router could judge message is from "lower" router and it should become the router of emergency transmission route. Thus, it will do as following: 1) Change state from "Normal" to "Detection". 2) Revise received emergency detection message. "Upper" field is revised to its "upper" router. "Sender" field is revised to its own id. 3) Broadcast the revised message to neighbors. If different, it means message is not from "lower" router. Therefore, it only changes state from "Normal" to "Suppressed" and suppresses this message. It becomes one router of barrier. Via the transmission of emergency detection message from emergency detected router until monitor station, emergency transmission route and barrier are constructed. Each router should be at only one of the following states:

- Detection: Forward received emergency information messages from "lower" router, suppress received normal sensing information message; 
- Suppressed: Suppress received emergency information messages, normal sensing information message, normal sensing information message from its connected router and routing messages;

- Normal: Forward received normal sensing information from connected sensors and normal sensing information messages from "lower" router; forward routing messages.

After transmission of emergency detection message, each community member knows which sensor sensed emergency and which router connects this sensor from "Source Sensor" field and "Source Router" field of received emergency detection message. All "Detection" members consist emergency transmission route. All "Suppressed" members consist barrier. Therefore, via the transmission of emergency detection message, community is constructed. Fig. 4 shows the sequence.

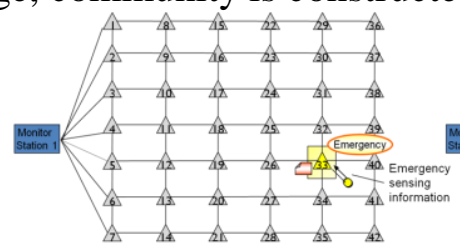

(a)

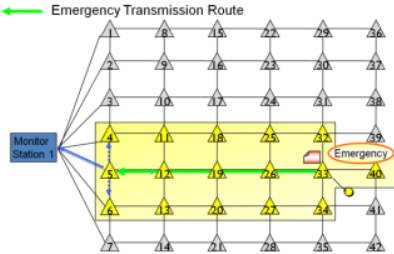

(c)

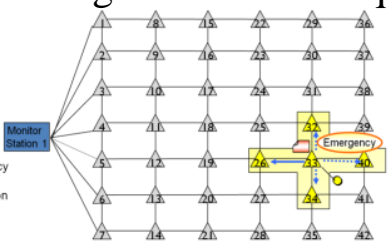

(b)

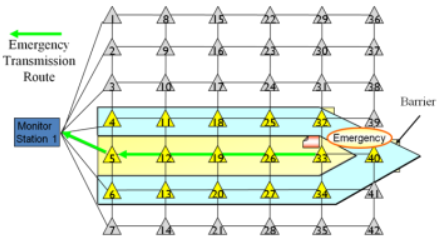

(d)

Figure 4. Emergency Transmission route and Barrier Construction

\section{Autonomous Alternative Route Searching}

When one monitor station received emergency detection message, it should generate alternative route search message which $\mathrm{CC}$ is "Alternative" as shown in Fig.5. "Sender" field of this message is the monitor station's id and the id should be inserted into "Route" field as start point of one alternative route. "Source Community" field is same as "Source Router" field of received emergency detection message. "Overlap" field is empty. Then the monitor station should broadcast the generated message to network.

\begin{tabular}{|l|l|l|l|l|l|}
\hline Alternative & Sender & Source Community & Route & Overlap & Msg id \\
\hline
\end{tabular}

Figure 5. Alternative Route Search Message

If received the message, router at "Suppressed" state should check whether message is for same community by "Source Community" field of message. If information is same as previously received emergency detection message's "Source Router" field, router should insert own id to the "Route" field of message and forward it. If different, the router should suppress this message.

Router at "Detection" state should suppress this message. Router at "Normal" state should only suppress this message. However, through the information of "Sender" field of this message, this "Normal" router could know which neighbor is at "Suppressed" state. 


\section{Autonomous Real-time Transmission for Multiple Emergencies}

Because overlap between communities is permitted in this subsection. Hence, if new emergency appears outside existed community, via emergency transmission route and barrier construction through this route, new community is constructed. Its emergency transmission route has no common part with existed community's emergency transmission route. And it is also not adjacent to existed community's emergency transmission route. After this community is constructed, monitor station will submit corresponding alternative route search message. If router of overlap part received this message, it should insert its id to "Route" and "Overlap" field of this message and forward it. Via transmission of this message, each "Detection" router which received this message could know alternative routes' information and overlap part. Fig. 6 shows the result.

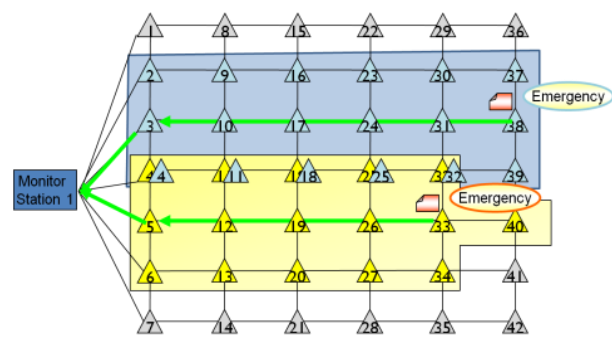

Figure 6. Autonomous Real-time Transmission for Multiple Emergencies

\section{Evaluation}

Simulator Omnet[10] is utilized for evaluation. 4D mesh network topology is utilized. Arrival rate of emergency information is the performance to evaluate.

Comparison is made between: 1)autonomous real-time transmission technology(ARTT); 2) congestion avoidance approach: Priority-based Hybrid Protocol (PHP)[3]; 3) Priority based transmission: Sequential Assignment Routing (SAR)[11].

In (a) of Fig.7, frequency of normal sensing information messages is $0.2 \mathrm{~Hz}$. ARTT achieved best performance of real-time property. There is one emergency in(b), 4 emergencies in (c) and 8 emergencies in (d). ARTT also achieve best performance as the emergency information's transmission is protected by ARTT but not protected by PHP and SAR.

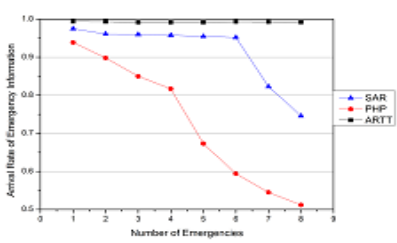

(a)

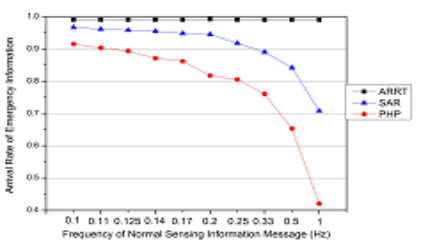

(c)

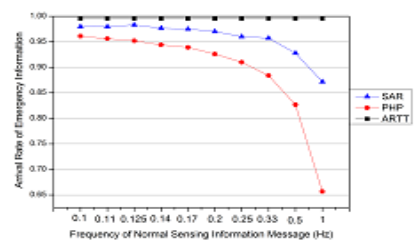

(b)

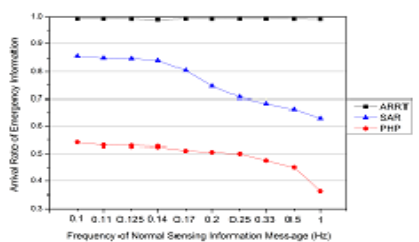

(d)

Figure 7. Evaluation Result 


\section{Conclusions}

Via proposed technology, when emergency happens, community which is composed of main route and barrier is autonomously constructed. Routers of emergency transmission route only transmits emergency information. Routers of barrier protect the transmission of emergency information from influence of normal sensing information's transmission. The efficiency of this technology has been confirmed by the result of evaluation.

\section{Acknowledgement}

This research was financially supported by the special scientific research fund of the Education Department of Shaanxi Province (No. 16JK1596) and Scientific and technological projects of Shaanxi Province (No.2015GY102).

\section{References}

[1] IF. Akyildiz, W. Su, Y. Sankarasubramaniam and E. Cayirci, Wireless sensor networks: a survey, Computer Networks, Vol. 38 pp. 393-422, 2002.

[2] K. Lorincz, D.J. Malan, T.R.F. Fulford-Jones, A. Nawoj, A. Clavel, V. Shnayder, G. Mainland, M. Welsh and S. Moulton, Sensor networks for emergency response: challenges and opportunities, IEEE Pervasive Computing, Vol. 3, Issue 4, pp. 16 - 23, 2004.

[3] Hsu-Jung Liu, Mei-Wen Huang, Wen-Shyong Hsieh and Jan C.J, Priority-Based Hybrid Protocol in Wireless Sensor Networks, HPCC 2009, pp. 214 - 221, 2009.

[4] A. Boukerche, R.B. Araujo and L Villas, A Wireless Actor and Sensor Networks QoS-Aware Routing Protocol for the Emergency Preparedness Class of Applications, IEEE Conference on Local Computer Networks 2006, pp. 832 - 839, 2006.

[5] K. Mori, Autonomous decentralized systems: concept, data field architecture and future trends, ISADS 1993, pp. 28-34, 1993.

[6] K. Mahmood, X. Lu, Y. Horikoshi, K. Mori, Autonomous Pull-Push Community Construction Technology for High-Assurance, IEICE TRANS. INF. \& SYST., Vol. E92-D. No.10 pp.1836-1846, October 2009.

[7] S. Niki, S. Murakami, K. Mahmood, X. Lu and K. Mori, Autonomous Decentralized Community Wireless Sensor Network Architecture to Achieve High-Speed Connectivity under Dynamical Situation, ISADS 2009, pp. 297-304, 2009.

[8] S. Vakil and B.Liang, Balancing Cooperation and Interference in Wireless Sensor Networks, SECON 2006, Vol. 1 pp. 198-206, 2006.

[9] C. Intanagonwiwat, R. Govindan, J. Heidemann, F. Silva, D. Estrin, Directed diffusion for wireless sensor networking, IEEE/ACM Transactions on Networking, Vol. 11, Issue 1, Feb. 2003, pp. 2-16

[10]http://www.omnetpp.org/ 
[11]K. Sohrabi, J. Gao, V. Ailawadhi and G. J. Pottie, Protocols for Self-Organization of a Wireless Sensor Network, IEEE Personal Communications, Vol. 7, Issue 5, pp. 16-27, Oct. 2000

[12]F.Wei, Y.Kotake, Md.E.Haque, Y.Fukunaga, T.Gouda, X.Lu, K.Mori, Autonomous Community Adjustment Technology for Emergency Management System, IEEE 10th International Symposium on Autonomous Decentralized System (ISADS 2011), pp.240-247, June 2011, Kobe, Japan 